\title{
MODELACIÓN MATTEMÁTICA DE UNA OPERACIÓN AÉREA
}

\section{MATHEMATICAL MODELLING OF AN AIR OPERATION}

rev.ciencia.poder.aéreo.7: 26-31, 2012

Autores
Diego Gerardo Roldán Jiménez
1

Jorge Mauricio Ruiz²

Fecha de recepción: 25 de abril de 2012

Fecha de aceptación: 13 de Julio de 2012

\section{Resumen}

El siguiente artículo da a conocer una modelación matemática con relación a una operación aérea donde se debe destruir un objetivo en tierra. En particular, el objetivo por considerar dispone de baterías antiaéreas suficientes para defenderse de un ataque aéreo. Se desarrollarán modelos probabilísticos para describir la situación, donde se pretende determinar la cantidad de aeronaves que se deben utilizar para maximizar la probabilidad de éxito y a su vez minimizar la cantidad de aeronaves aliadas derribadas.

Palabras clave: Bombardeos a objetivos estáticos, modelación matemática.

\section{Abstract}

This article presents how a mathematical model applied to an air operation where ground targets are being attacked. Particularly, it considers having enough anti aircraft batteries to be defended from an air attack. It develops probabilistic models to describe the situation, trying to determine the quantity of airships to be used to maximize the probability of success and at the same time, to minimize the quantity of downed allied airships.

Key words: Static Objects Bombing, mathematical modeling. 


\section{Introducción}

La situación que se considera es la destrucción total de un objetivo en tierra, que se encuentra bien defendido por baterías antiaéreas. Para ello, se deben enviar un número determinado de aeronaves, que garanticen el éxito de la misión. El problema inicial que se enfrenta es que las aeronaves van a recibir fuego enemigo desde tierra, lo cual puede ocasionar daños inminentes y quizá el derribo de estas aeronaves. Por ello, el factor inteligencia es muy importante en esta operación, ya que permitirá determinar con cuales tipos de defensas antiaéreas cuenta el enemigo, la cantidad y la eficiencia de estas máquinas de defensa. Las defensas antiaéreas en su intento de defender el objetivo atacarán constantemente a las aeronaves involucradas en la misión con el fin de derribarlas. A las defensas aéreas se les acostumbra a llamar SAM (por sus siglas en ingles Surface Air Missile) en este modelo estas defensas antiaéreas son automáticas y como tal reaccionan ante un posible ataque enemigo. La probabilidad de acertar de estas armas depende de la pericia del que las manipula y de la tecnología con la que cuente.

\section{Método}

Para el escenario donde se esté lanzando determinado número de munición desde tierra, es conveniente utilizar una distribución de probabilidad que indique el fracaso o el éxito a la hora de atacar aeronaves enemigas.

En la primera parte, se encuentra la distribución de probabilidad binomial con algunas de sus propiedades y se menciona la noción de simulación analítica para modelar una situación que involucre más variables.

\subsection{Una Operación Aérea Planificada}

El centro de operaciones aéreas estratégicas, planea un ataque a un objetivo que se encuentra muy bien defendido, el centro dispone de bombarderos de gran altitud para realizar la misión. Por problemas de seguridad nacional se debe asegurar el éxito de la operación durante el primer día de ataque.

Pregunta : ¿Cuántos bombarderos deben enviarse a este objetivo, con el fin de garantizar su total destrucción?

Esta es la pregunta típica que se realiza en el centro de operaciones estratégicas. Para plantear su solución, se necesita saber las condiciones, bajo las cuales se enviarán aeronaves a la misión y por ello, los informes de inteligencia son muy importantes para identificar mejor al objetivo. Normalmente, estos informes de inteligencia traen las coordenadas donde se planea el ataque, el terreno y la dimensión de objetivo (Área).

Esta información ayudará a determinar el tipo de bombas que deben ser utilizadas durante el ataque. En este tipo de operaciones el daño colateral debe ser mínimo y en consecuencia está información es muy importante.

Con la información suministrada anteriormente, inteligencia puede realizar estimaciones sobre la efectividad de los bombarderos y determinar la probabilidad de éxito individual. Aquí también es importante contar con aeronaves de alta tecnología, pues mientras se disponga de más tecnología mejor será esta probabilidad. Además, teniendo en cuenta las características de las aeronaves, también se pueden hallar cifras que indiquen la probabilidad de atravesar las defensas aéreas y lograr destruir el objetivo. Para fines prácticos se considera que la misión es exitosa si se logra una efectividad mayor o igual a $99 \%$.

Otro dato fundamental que debe suministrar inteligencia, es el qué tipo de defensa, de la que dispone el objetivo, esta información debe ser precisa y detallada, porque permitirá ajustar las cifras para determinar la probabilidad de que alguna de estas baterías antiaéreas impacte a una aeronave.

Para empezar a describir el modelo, se necesita hallar una expresión que determine la probabilidad de que la misión sea un éxito. Se considera que el número de aeronaves derribadas será una variable aleatoria $X$ cuya distribución se mencionará más adelante. También se define $X$, como la expresión que representa la probabilidad $P_{1}$ de éxito de la misión dado que $X=i$ aeronaves han sido derribadas, antes de que puedan atacar el objetivo, y se necesita fijar la probabilidad de que $X=i$ aeronaves sean derribadas.

En esta expresión $X_{1}$ se asigna a la distribución de $X$ con el fin de determinar el valor esperado de éxito de $i$ aeronaves dado que $x=i$ aeronaves han sido derribadas. De esta forma, se define a una nueva variable $S$ como la probabilidad de éxito de la misión:

$$
S=\sum_{i} X_{i} P(X=i) \quad i=1,2, \ldots, m
$$

donde $m$ es la cantidad de misiles lanzados desde tierra. Para determinar los valores que toma, observemos que si $N$ aeronaves son enviadas a la misión y $x=i$ aeronaves son destruidas antes de completar la misión, entonces $N$ - iaeronaves atacan el objetivo. Si es la probabilidad de que una aeronave destruya el objetivo, 1 $P$ es la probabilidad de que fracase.

Ahora se supone que la variable aleatoria que denota el número total de aeronaves destruidas es una variable aleatoria con distribución binomial; es decir:

$$
P(X=i)=\left(\begin{array}{c}
m \\
i
\end{array}\right) q^{i}(1-q)^{m-i} \quad i=1,2, \ldots, m
$$

donde $m$ es el número de misiles lanzados y $q$ es la probabilidad de que un misil derribe una aeronave. 
En la expresión (1) los valores desconocidos son $m$ y $N$. Para conocer el valor de $m$ se debe determinar el tiempo que las aeronaves se encuentran expuestas al fuego enemigo, ya que las baterías antiaéreas usualmente lanzan una determinada cantidad de misiles en un tiempo dado. V Se debe considerar la velocidad a la que vuelan las aeronaves y el rango de alcance efectivo de cada SAM, luego el tiempo que las aeronaves permanecerán expuestas mientras realizan el ataque está dado por:

$$
t=\frac{A}{v_{0}}
$$

donde $A$ es el alcance del radar de las defensas antiaéreas y $v_{0}$ es la velocidad a la cual están volando las aeronaves. Se plantea el siguiente objetivo:

\section{Objetivo}

Hallar el valor mínimo de $N$ tal que $S>0.99$

\section{Un Ejemplo Ideal}

Hay que destacar que algunas probabilidades en estas operaciones, como por ejemplo la probabilidad de detección y destrucción del objetivo depende del armamento que se está utilizando. Estos cálculos de efectividad de destrucción de las bombas, son calculados por la casa fabricante y normalmente dependen del ángulo de incidencia entre el objetivo y la aeronave atacante.

Es posible encontrar características sobre baterías antiaéreas en diferentes sitios de casas fabricantes. En el siguiente ejemplo (Meerschaert M.) existe una situación específica, donde el trabajo de inteligencia suministra información detallada de las capacidades del objetivo y capacidades propias de las aeronaves a utilizar en la misión (Ver Ejemplo 1).

Los datos de inteligencia suministrados, informan que el objetivo está defendido con dos baterías antiaéreas y suficientes armas de corto alcance; cada batería antiaérea tiene su propio sistema de guía y rastreo, el cual es capaz de rastrear dos aeronaves con alcance de $80 \mathrm{~km}$, también es capaz de guiar dos misiles a la vez con un rango efectivo de radar de $24 \mathrm{~km}$. Cada misil viaja a una velocidad de $1600 \mathrm{~km}$ por hora. Se estima que la probabilidad de que un misil impacte una aeronave es del $60 \%$ y se sabe que puede disparar 1 misil cada 30 segundos; además cada SAM dispara una sola vez a cada aeronave.

Por otra parte, los bombarderos viajan a una velocidad de 804 kilometros por hora, a una altitud de $8 \mathrm{~km}$ y el ataque requiere una permanencia en el área de 1 minuto.
Ejemplo 1. El jefe del centro de operaciones aeronáuticas,

sabe que las aeronaves que enviará a la misión tienen una probabilidad individual de destruir el objetivo del 50\%, asumiendo que atraviesan las defensas aéreas y encuentran al objetivo. La probabilidad de que cada aeronave adquiera el objetivo es del $90 \%$.
En este sentido, se quiere determinar el tiempo total al que se expondrá cada aeronave; para ello se determina el alcance efectivo del radar y la velocidad de la aeronave reemplazando en la expresión (2):

$$
t_{0}=\frac{24 \mathrm{Km}}{804 \mathrm{Km} / \mathrm{h}} \frac{60 \mathrm{~min}}{1 \mathrm{~h}}=1.8 \mathrm{~min}
$$

A este valor se le debe sumar el minuto de permanencia mínimo que informa el plan de vuelo, así el tiempo total de exposición será aproximadamente de 3 minutos.

Como las dos baterías antiaéreas pueden lanzar 2 mísiles cada 30 segundos, entonces se concluye que el número total de mísiles en el aire durante la operación será de $m=10$ mísiles. Para solucionar el modelo se harán simulaciones por computadora con las siguientes entradas:

$$
\begin{aligned}
& m=10 \\
& p=(0.9)(0.5) \\
& q=(0.6)
\end{aligned}
$$

y para diferentes valores de $N$. Se ilustra los resultados en la Figura 1.

Un mínimo de $N=15$ aeronaves son necesarias para asegurar el éxito de la misión. Un número bastante considerable sobre todo si se cuenta con un poder aéreo lo suficientemente limitado. Se necesita hacer un análisis de sensibilidad para tener una visión más amplia, de cuál será el número indicado de bombarderos para realizar esta misión. 
Primero se considera la probabilidad de éxito $S=0.99$. Este es un valor conveniente a variar. En la Figura (2) se muestran los efectos de hacer esta variación. Se observa que $N=15$ es una buena decisión, aunque un número de aeronaves entre 10 y 20 sería aceptable. Más de 20 aeronaves es innecesario, ya que no hay una variación considerable de la probabilidad de éxito.

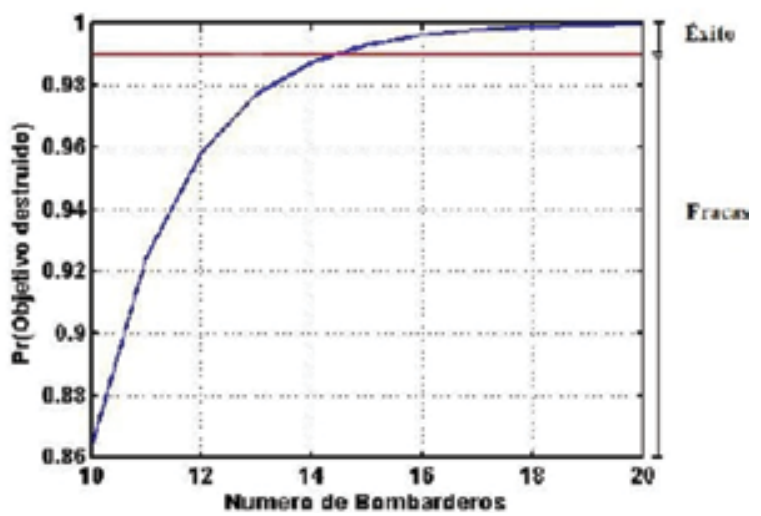

Figura 1: La probabilidad $S$ de éxito y los bombarderos $N$ enviados.

\subsection{El Mal Clima}

Cuando se planea una operación aérea, entre los elementos más importantes que se deben considerar es el clima. Si hay buen clima, la probabilidad de que un bombardero pueda destruir el objetivo será mayor, debido a que son más fáciles las condiciones de vuelo. Normalmente, antes de lanzar un ataque aéreo se espera el reporte de meteorología aeronáutica, con el fin de determinar si es viable o no el ataque. En el modelo propuesto, el mal clima se verá reflejado en la probabilidad $p$, de que un bombardero destruya el objetivo.

Para este caso particular por, tiene que $p=(0.9)(0.5)$ y si la probabilidad de detección se reduce a la mitad, es decir $p=(0.5)$ (0.5) y si $S>0.99$, entonces se necesitarán $N=23$ aeronaves. De igual forma, si la probabilidad de detección ahora es 0.3 , entonces $N=35$ aeronaves son requeridas. La relación que hay entre la probabilidad de detección y el número de aeronaves requeridas para la misión se ilustran en la Figura 3.

Es obvio que no se quiere volar la misión del ejemplo con mal clima. En la Tabla 1 se muestra que el ejemplo con los parámetros dados, es bastante sensible cuando la probabilidad de detección está entre 0.2 y 0.3 , caso opuesto a lo que sucede para valores entre 0.8 y 0.9 . Esto es porque la cantidad de aeronaves se incrementa en un factor de 13 aeronaves por cada $10 \%$ de probabilidad de detección.

\subsection{Tecnología Disponible}

Una consecuencia del modelo es analizar el impacto operacional que traen los avances tecnológicos. Por ejemplo, si se disponen de bombarderos que viajen al doble de la velocidad, estos reducirín el tiempo de exposición a 15 segundos.

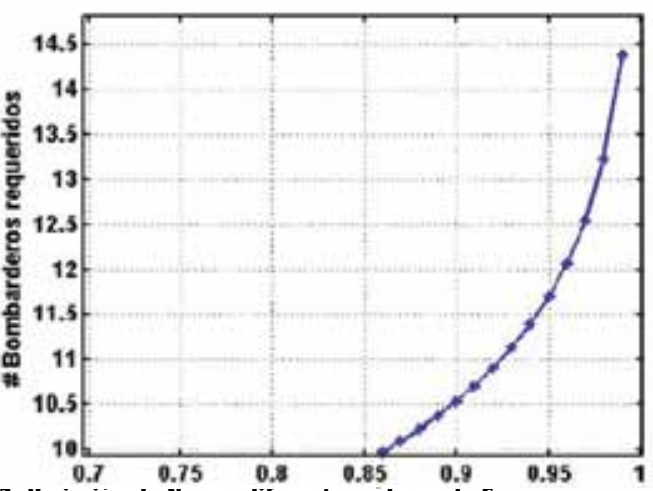

Figura Z: Variacion de N para diterentes valores de S.

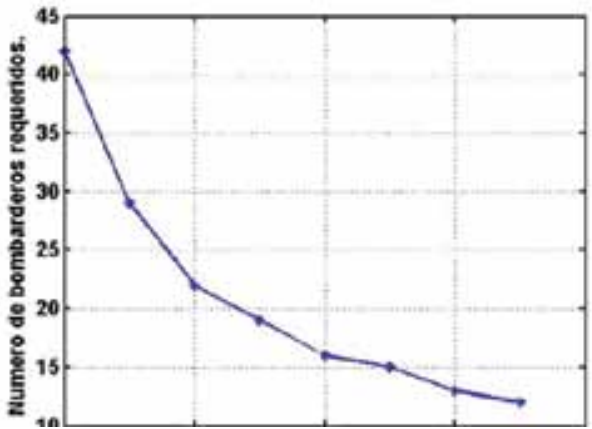

Figura 3: Número de bombarderos contra la probabilidad de detección de las aeronaves.

Por lo tanto, en la operación de estudio las aeronaves se verán expuestas al fuego antiaéreo solo por un 1 minuto, donde las baterías SAM lanzarán un total de $m=4$ misiles. Con estas condiciones solo se necesitarán $N=11$ aeronaves para garantizar un éxito de la misión $S$ mayor al $99 \%$.

La Figura 4 muestra la relación que hay entre el número de bombarderos enviados y la probabilidad de éxito de la misión en el caso de que se lancen $m=10$ misiles (trayectoria verde) y en el caso del concepto avanzado de aeronave $m=4$ (trayectoria azul). Para una comparación de referencia incluimos el escenario donde no se lanzan misiles $m=O$ (trayectoria amarilla.

En la Figura 5 se observa que a mayor cantidad de aeronaves en el aire y mayor probabilidad de impacto de los misiles, mayor será el número de aeronaves derribadas.

\subsection{Aeronaves Derribadas}

Cuando las aeronaves cumplen su misión y se preparan para regresar a la base, ellas aún están expuestas a las baterías antiaéreas, entonces, la siguiente pregunta es:

Pregunta: ¿Cuántas aeronaves se perderán en toda la misión? 
Tabla 1: Sensibilidad entre los valores de p y N

\begin{tabular}{ccc}
\hline Valores de & Valores de & Sensibilidad \\
\hline $0.2-0.3$ & $42-29$ & 1.3 \\
\hline $0.3-0.4$ & $29-22$ & 0.7 \\
\hline $0.4-0.5$ & $22-19$ & 0.3 \\
\hdashline $0.5-0.6$ & $19-16$ & 0.3 \\
\hline $0.6-0.7$ & $16-15$ & 0.1 \\
\hline $0.7-0.8$ & $15-13$ & 0.2 \\
\hline $0.8-0.9$ & $13-12$ & 0.1 \\
\hline
\end{tabular}

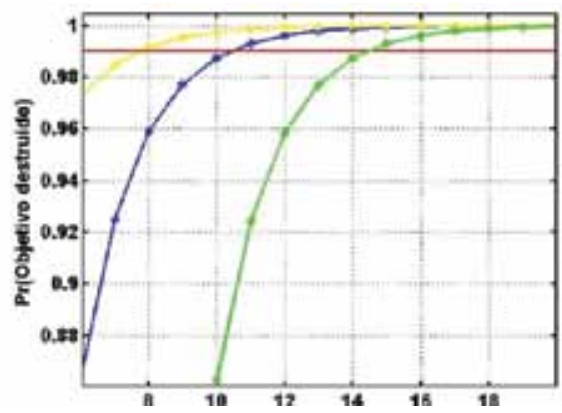

Figura 4: Éxito de la misión $\mathrm{S}$ y el número de bombarderos enviados $\mathrm{N}$. Comparación de los tres casos $\mathbf{m}=\mathbf{0 , 4} \mathbf{y} 10$.

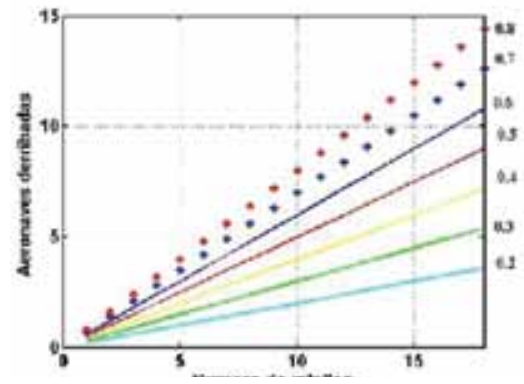

Figura 5: Relación entre cantidad de misiles en el aire y aeronaves derribadas para diferentes valores de $\mathbf{q}$.

Para conocer esta cifra se define la siguiente expresión:

$$
\text { Lost }=\sum_{i} i P(X=i) \quad i=1,2, \ldots, m
$$

donde $i$ representa el número de aeronaves impactadas por los misiles y $p(x=i)$ representa la distribución binomial. Así, con el minuto adicional de permanencia, se tiene que las aeronaves deben permanecer por lo menos 4.6 minutos en la zona de exposición. En este tiempo cada batería antiaérea lanza 9 misiles; así habrá $m=18$ misíles en el aire. Luego: $m=18$

$p=(0.9)(0.5)$

$q=0.6$

$N=15$

$Y$ se obtiene que en total se derriban 11 aeronaves. Un resultado trágico para la operación, esto es porque el valor unitario de un bombardero económico, (por ejemplo el Supertucano) es de varios millones de dólares. En este momento, se debe considerar la posibilidad de permanecer menos tiempo en la zona de exposición, para ello, se modifican los tiempos de entrada, permanencia y salida.

El tiempo de permanencia en la mayoría de los casos es inalterable, aquí; es importante disponer de aeronaves más veloces. Si ahora en el ejemplo se consideran aeronaves que vuelan a una velocidad de 1.930 kilómetros por hora (bombarderos supersónicos) y solo permanecen en el área de ataque por 15 segundos ( 0.25 minutos) se tiene que gastarán 0.75 minutos entrando en la zona de exposición y 0.75 minutos saliendo, por lo cual gastarán un total de 1.75 minutos. Este tiempo suficiente para que las baterías antiaéreas lancen un total de $m=6$ misíles. Así:

$m=6$

$p=(0.9)(0.5)$

$q=0.6$

$N=15$

y solo se tendrán 4 aeronaves derribadas. Por lo tanto, la ventaja tecnológica es de gran ayuda en estas operaciones.

\section{Conclusiones}

El modelo mostrado es bastante restringido en el sentido de que consideran valores ideales e hipotéticos en una operación aérea simulada. Estos modelos se simplifican al permitir independencia de eventos, como es el caso de asumir la misma probabilidad de destrucción por cada una de las aeronaves involucradas en la misión. En realidad esta independencia no sucede porque la probabilidad de detección de cada una de las aeronaves disminuye cuando cae una bomba sobre el objetivo, ya que genera polvo y humo, esto afecta la efectividad de estas armas. Aunque al realizar un análisis de sensibilidad sobre este parámetro se observa que el número de aeronaves no cambia de manera significativa.

El modelo permite incorporar una distribución de probabilidad a la variable que describe la cantidad de aeronaves derribadas. Debido la naturaleza de la situación, una distribución adecuada para este modelo es la distribución de Binomial que describe la probabilidad de éxitos en intentos. De esta forma, se facilita describir el modelo utilizando simulación analítica y posteriormente un posible desarrollo analítico con la ayuda del binomio de 
Newton. La simulación analítica en donde se usa la distribución de Binomial, no permite la situación donde se presentan más misiles en el aire que aeronaves, esto es por que la variable que describe el éxito de la operación toma valores positivos.

En situaciones de guerra donde se quiere debilitar al enemigo, es evidente el deseo de desarticular sus principales fuentes de combate(por ejemplo, pistas, fábricas, artillería, etc...) Estos objetivos estratégicos, se espera sean muy bien defendidos por el rival, ya sea por tropas en tierra o como sugiere el modelo a través del uso de baterías antiaéreas. El factor inteligencia permite describir cómo está planteada la defensa enemiga. La destrucción de estas fortalezas rivales pueden cambiar el curso de un conflicto armado, permitiendo quizá el fin del mismo. De esta forma, una versión más avanzada del modelo puede ayudar a simular operaciones reales entre distintas fuerzas, ya sea para ejercicios técnicos de combate o para complementar una eventual operación que esté involucrada en una hipótesis de guerra. De manera semejante, este modelo puede validarse con operaciones hechas anteriormente en el territorio nacional parametrizando las probabilidades de éxito, de las cuales dispone el armamento aéreo y las aeronaves de combate de la FAC utilizadas usualmente para operaciones que involucren bombardeos.

\section{Referencias}

Barnier, W. (s.f.). Expected Loss in Keno. Módulo 574: UMAP.

Bender, E. A. (s.f.). An introduction to Mathematical Modeling. New York: Wiley \& Sons.

Billingsley, P. (1979). Probability and measure. New York: Wiley \& Sons.

Carlson. : Conditional Probability and Ambiguous information. Modulo 391. UMAP.

Dym, C.L. (2004). Principles of Mathematical Modeling. Claremont, California: Academic Press.

Feller, W. (1971): An introduction to probability theory and its applications. Vol. 2. 2nd Ed. New York: Wiley \& Sons.

Meerschaert, M. (1993). Mathematical Modeling. New York: Academic Press, Inc.

Ross, S. (s.f.). Introduction to probability models. 3rd ed. New York: Academic Press Inc. 